\title{
Retrieving Temperature and Moisture Profiles from AERI Radiance Observations: AERIPROF Value-Added Product Technical Description
}

Revision 1

$\begin{array}{ll}\text { W.F. Feltz } & \text { D.D. Turner } \\ \text { H.B. Howell } & \text { W.L. Smith } \\ \text { R.O. Knuteson } & \text { H.M. Woolf } \\ \text { J. Comstock } & \text { C. Sivaraman } \\ \text { R. Mahon } & \text { T.Halter }\end{array}$

April 2007

Work supported by the U.S. Department of Energy,

Office of Science, Office of Biological and Environmental Research 


\section{DISCLAIMER}

This report was prepared as an account of work sponsored by the U.S. Government. Neither the United States nor any agency thereof, nor any of their employees, makes any warranty, express or implied, or assumes any legal liability or responsibility for the accuracy, completeness, or usefulness of any information, apparatus, product, or process disclosed, or represents that its use would not infringe privately owned rights. Reference herein to any specific commercial product, process, or service by trade name, trademark, manufacturer, or otherwise, does not necessarily constitute or imply its endorsement, recommendation, or favoring by the U.S. Government or any agency thereof. The views and opinions of authors expressed herein do not necessarily state or reflect those of the U.S. Government or any agency thereof. 


\begin{abstract}
This document explains the procedure to retrieve temperature and moisture profiles from high-spectral resolution infrared radiance data measured by the U.S. Department Of Energy (DOE) Atmospheric Radiation (ARM) Program's atmospheric emitted radiance interferometer (AERI) instrument. The technique has been named the AERIPROF thermodynamic retrieval algorithm. The software has been developed over the last decade at the University of Wisconsin-Madison and has matured into an ARM Value-Added Procedure. This document will describe the AERIPROF retrieval procedure, outline the algorithm routines, discuss the software heritage, and, finally, provide references with further documentation.
\end{abstract}




\section{Contents}

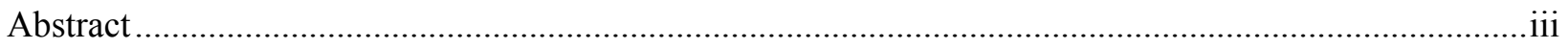

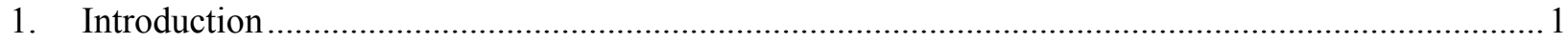

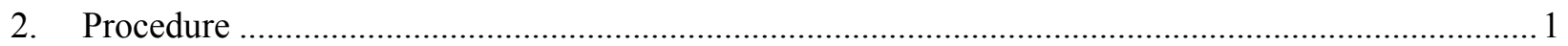

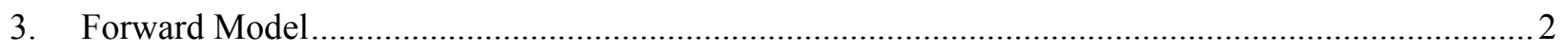

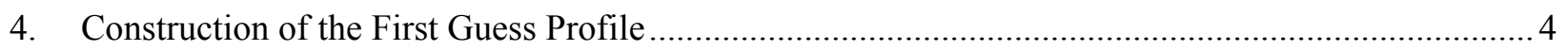

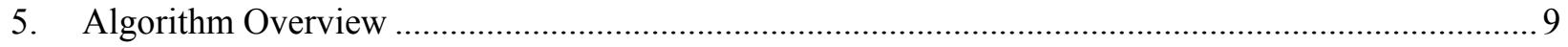

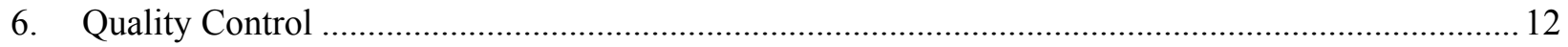

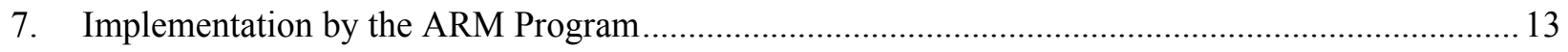

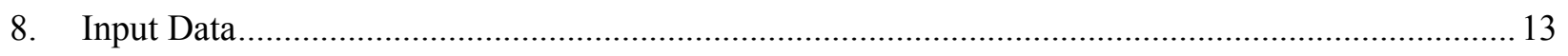

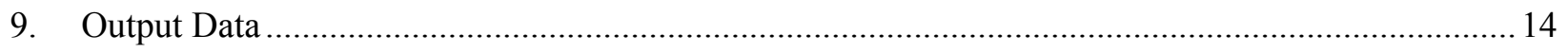

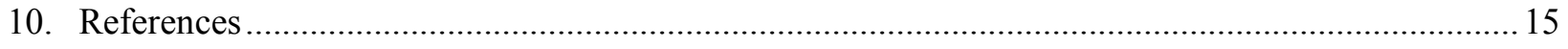

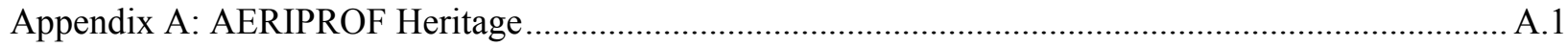

Appendix B: AERIPROF Subroutine Descriptions ..................................................................... B.1

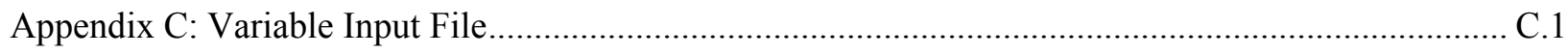

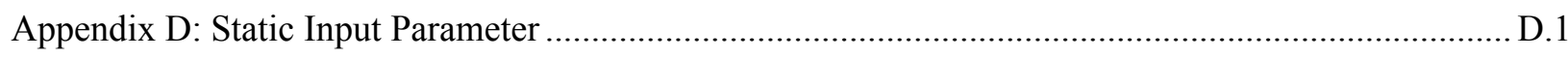

Appendix E: AERIPROF Algorithm Flowcharts.............................................................................

Figures

1 An example of an AERI infrared radiance measurement at one wavenumber resolution ............... 3

$2 \quad$ Weighting functions defined by ground-based AERI spectral regions ......................................... 4

3 Mean spectral differences between observed AERI radiances and fastmodel calculations using

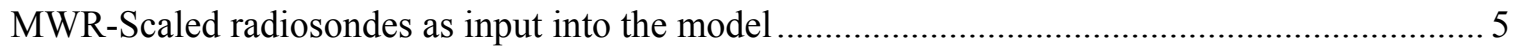

4 AERIPROF time-height temperature, moisture and data availability cross-sections indicating where RUC profiles and AERIPROF retrievals are available from the surface to 14 kilometers for

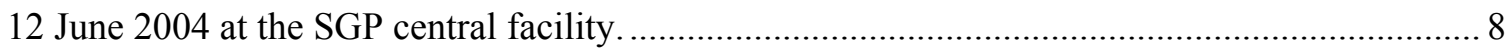

5 Same as Figure 4, but highlighting the lower troposphere

6 Several examples of AERIPROF temperature and water vapor retrievals compared to radiosonde

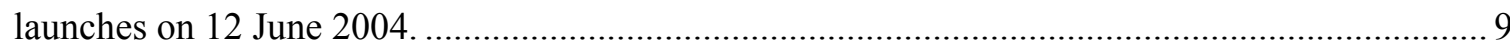




\section{Introduction}

One of the goals of the Atmospheric Radiation Measurement (ARM) Program is to collect a long-term series of radiative and atmospheric state observations to improve the parameterization of these processes in global climate models. The ARM Program intended to move away from the traditional approach of directly measuring profiles of temperature and moisture using radiosondes, which is expensive in terms of expendables and manpower, and develop methods to retrieve these profiles with ground-based remote sensors. The atmospheric emitted radiance interferometer (AERI), whose radiance data contains information on the vertical distribution of water vapor and temperature, is an integral part of the ARM profiling plan.

Profiles of temperature and water vapor have been retrieved from spectrally resolved infrared observations since the early 1970s. One of the first examples demonstrating this procedure was given by Smith (1970). Essentially, the radiative transfer equation is inverted to generate these desired profiles from the radiance observations. However, retrieving temperature and water vapor profiles (henceforth called T/q profiles) from radiance observations is an ill-posed problem. Therefore, a "first-guess" profile is used in an iterative procedure in the AERI T/q retrieval algorithm (henceforth called AERIPROF) to constrain the retrieval.

The AERIPROF algorithm has been developed and continually improved by scientists at the Cooperative Institute for Meteorological Satellite Studies (CIMSS) at the University of Wisconsin. The early history and background on the evolution of the technique is given by Feltz (1994), with a summary of the more recent developments given in Appendix A. Multiple papers have been published showing results from this algorithm (Feltz et al. 1998, Turner et al. 2000, Feltz et al. 2003, Feltz and Mecikalski 2002, Schmit et al. 2002). The basic steps and mathematics behind the physical retrieval are given in Smith et al.

(1999). This technical report provides additional details not given in these papers.

\section{Procedure}

The AERIPROF uses a physical retrieval approach, in which downwelling radiance is computed from an assumed atmospheric state and compared with the observed radiance; differences between the two are propagated backwards to modify the assumed T/q input profile. This procedure is continued until the radiance computed from the modified $\mathrm{T} / \mathrm{q}$ profiles matches the observed radiance within some tolerance. When this is achieved, the retrieval is said to have converged, and the modified T/q profile is the retrieved result.

The AERIPROF physical retrieval uses an "onion-peeling" approach, where the profiles are modified at the surface first, then the changes are made progressively higher in the atmosphere. Both the water vapor and the temperature profiles are retrieved in this manner simultaneously. This technique was chosen over other schemes, such as the optimal estimation approach (Rodgers 2000), because the weighting functions peak at or near the surface and, thus, the onion-peeling technique was the only approach that remained stable (W.L. Smith, personal communication, 2004). Details of the physical retrieval used in AERIPROF are given in Smith et al. (1999).

Two important components of the AERIPROF algorithm that need further explanation are the forward radiative transfer model and the logic used to determine the first-guess T/q profile. 


\section{Forward Model}

As indicated above, a forward radiative transfer model is needed in the physical retrieval to compute the radiance from the assumed atmospheric state. The forward model must be accurate, but the requirement for fast computation excludes the use of detailed line-by-line radiative transfer models.

The fast forward model used in AERIPROF is a variant of RTTOV (Eyre 1991, Garand et al. 2001). To generate the model, a line-by-line radiative transfer model is used to compute monochromatic optical depths for dry air, water vapor, and ozone for a set of specified atmospheric profiles on a fixed pressure grid. These monochromatic optical depths are converted to atmospheric transmittances, which are then convolved with the AERI's spectral response function to yield effective transmittances. The effective transmittances are then apodized (using Norton-Beer) to reduce the convolution-introduced "ringing." These convolved and apodized transmittances are then converted back into effective layer optical depths. Regressions are then used to relate these optical depths to the atmospheric state at each pressure level for each AERI spectral element. This process is performed for dry air (which includes all absorbing gases other than water vapor and ozone), water vapor, and ozone, and the regression coefficients are stored into binary coefficient files. The fast model then computes radiance by using these regressions to obtain the optical depths at each level and spectral element for the dry air, water vapor, and ozone; these optical depths are then combined for each layer and the radiative transfer calculation is completed.

The fast model used in the first two ARM versions of AERIPROF (called 1Feltz and 2Feltz, respectively) was based upon the Fast Atmospheric Signature Code (FASCODE; Clough et al. 1981) line-by-line radiative transfer model using the water vapor continuum absorption model CKD v2.1 (Clough et al. 1989). However, research by ARM in longwave radiative transfer modeling has resulted in a wellvalidated line-by-line model called LBLRTM (Clough et al. 1992, Clough and Iacono 1995, Turner et al. 2004), and thus the fast model in 3Feltz was built using the LBLRTM v6.01 and CKD v2.4 (information on the differences in LBLRTM / CKD versions can be found at http://rtweb.aer.com). It should be noted that the training dataset used to generate the regression coefficients includes 31 radiosonde-rocketsonde sets launched from the tropics to the Arctic, plus the 1976 U.S. Standard Atmosphere.

The algorithm does not use the entire spectrum for the physical retrieval, as there is little information on the vertical structure of the water vapor and temperature in some spectral regions. Instead, regions of the spectrum are used that have the most information on this vertical structure with as little interference from other gases as possible. Thus, spectral regions near the $v_{2}(15 \mu \mathrm{m})$ and $v_{3}(4 \mu \mathrm{m}) \mathrm{CO}_{2}$ absorption bands are used in the temperature profile retrieval, while spectral regions on the wings of the rotational and $v_{2}$ $(6.6 \mu \mathrm{m})$ water vapor absorption bands are used for the water vapor profile retrieval. These spectral regions are shown in Figure 1. The weighting functions depicting the altitudes where largest infrared energy contribution is occurring are shown in Figure 2. 

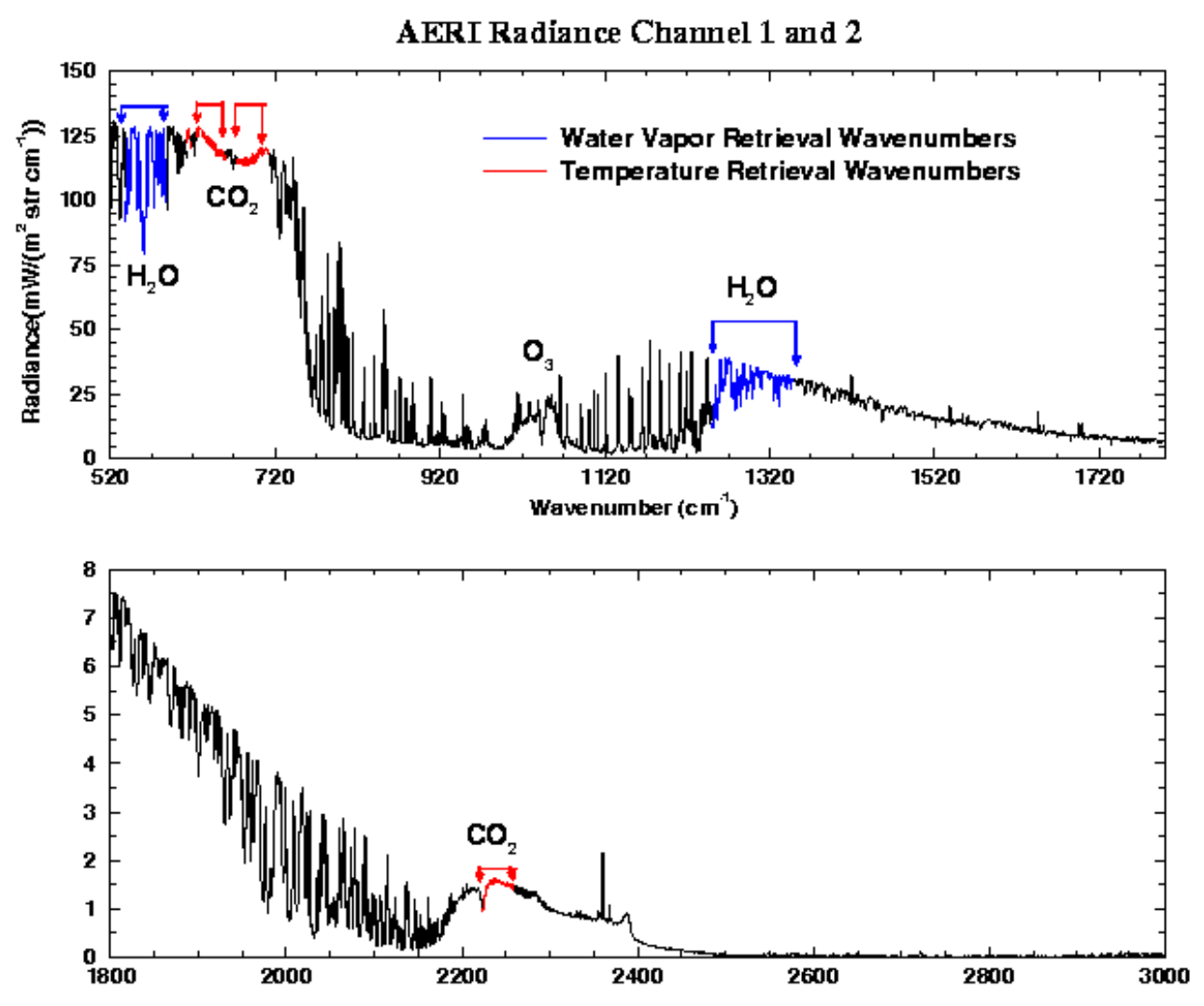

Figure 1: An example of an AERI infrared radiance measurement at one wavenumber resolution. The colored regions indicate the part of the spectrum in which temperature (red) and water vapor (blue) profiles are derived using the AERI physical retrieval algorithm.

Regression uncertainties and spectroscopic errors in the forward model require that a bias error spectrum be used to achieve closure between the observed and calculated radiance spectra. Operationally, the static bias spectrum is subtracted from the observed spectrum before each retrieval. The bias spectrum is determined offline by selecting a suite of radiosondes and using the fast model to compute the downwelling radiance. The bias spectrum is then computed as the mean difference between the coincident AERI observations and these calculations. Care needs to be taken to ensure that the radiosonde profiles used in the determination of the bias spectrum accurately represent the atmosphere (e.g., do not have a dry bias in the water vapor observations), otherwise the radiosonde errors will be introduced into the retrieval. The extensive work performed under the auspices of the ARM Water Vapor Intensive Operational Periods (WVIOPs) has shown that microwave radiometer (MWR) scaled radiosonde humidity profiles have less bias and variability in their water vapor calibration than the nominal unscaled radiosonde profiles (Revercomb et al. 2003, Turner et al. 2003). Therefore, MWRscaled radiosondes from the LSSONDE value-added procedure (VAP) are used to determine the spectral bias used in the AERIPROF VAP. Six clear-sky profiles were used to compute the bias spectrum. The profiles were from May 1996 during conditions with average precipitable water vapor (PWV) for the SGP region $(\sim 2 \mathrm{~cm} P W V)$. No strong vertical gradients in the temperature or water vapor in the boundary layer existed. The resulting bias spectra for the 1Feltz / 2Feltz (based upon FASCODE) and 3Feltz (based upon LBLRTM) algorithms are shown in Figure 3. Note the improvement in the absorption line parameters in the HITRAN database and the other improvements in the LBLRTM have resulted in a significantly smaller bias spectrum. In fact, because the bias spectrum is close to zero and spectrally flat 
in both the $v_{2}$ and $v_{3} \mathrm{CO}_{2}$ bands, AERIPROF 3 Feltz does not apply a spectral bias for the $\mathrm{T}$ retrievals (although a bias is still applied for the retrievals of q). The goal is to minimize, and eventually remove, the bias spectrum as the spectroscopy and forward model construction improve.

The radiosondes and associated fast-model calculations are also used to compute an observed-versusmodel difference (RMS) spectrum. This RMS spectrum is used to determine when the retrieval has converged.
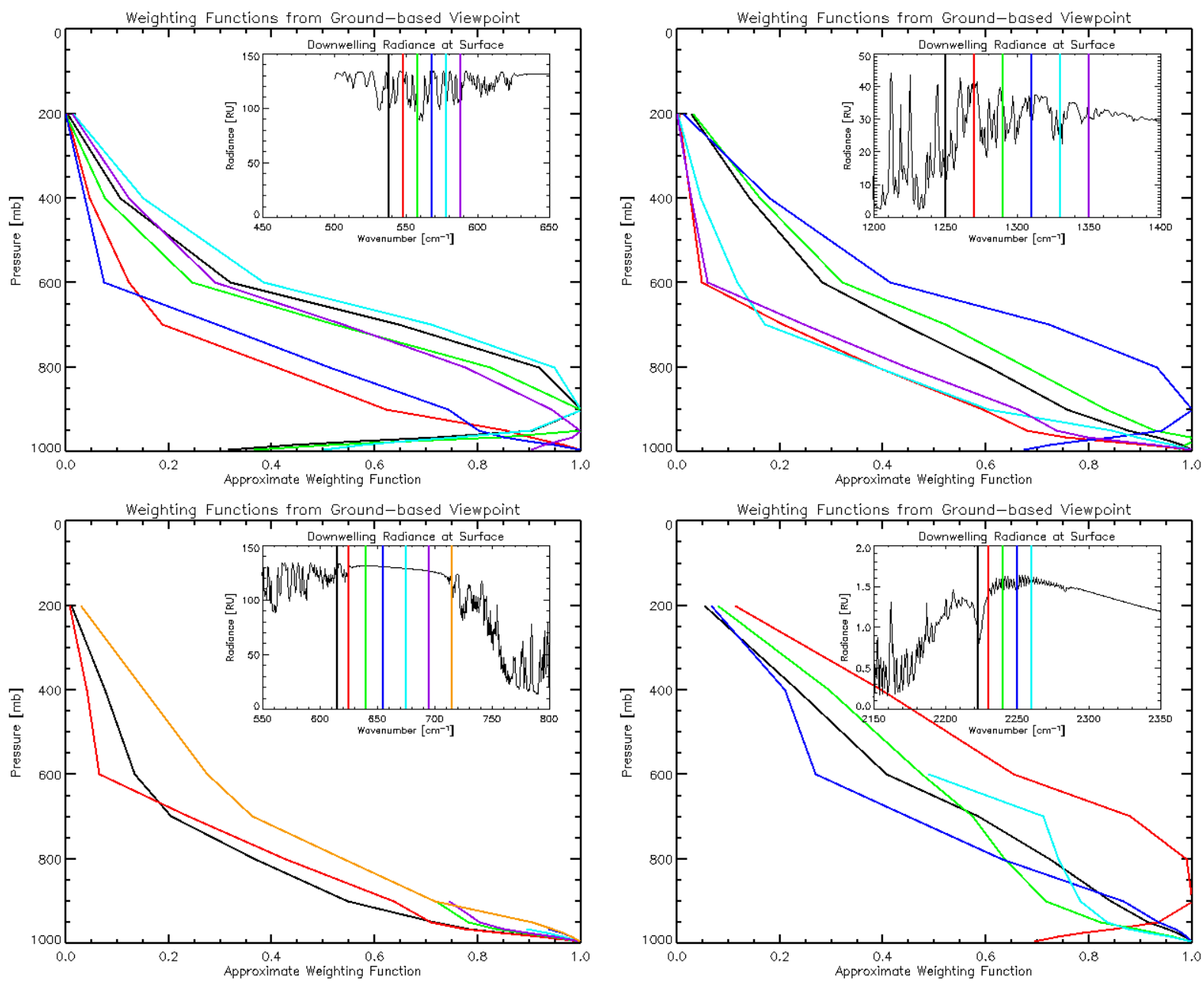

Figure 2: Weighting functions defined by ground-based AERI spectral regions (shown in upper righthand corner of images). The two upper panels show example weighting functions for water vapor sensitive spectral regions while the two lower panels provide similar examples for temperature sensitive spectral regions used in AERIPROF.

\section{Construction of the First-Guess Profile}

As indicated above, the AERIPROF needs a first guess of the atmospheric state. This first guess can be a radiosonde profile, satellite retrieved profile, a profile from a numerical weather prediction model, or some combination of the above. Early versions of the code (before 1992) used a radiosonde formatted into a BRF (binary record format) file as the first guess; this option is still available in the algorithm by setting the input flags appropriately. However, to make the code operational for ARM, especially at the 
SGP boundary facilities where radiosondes are not launched regularly, a statistical retrieval was developed to derive a first-guess profile directly from the observed radiance spectrum.
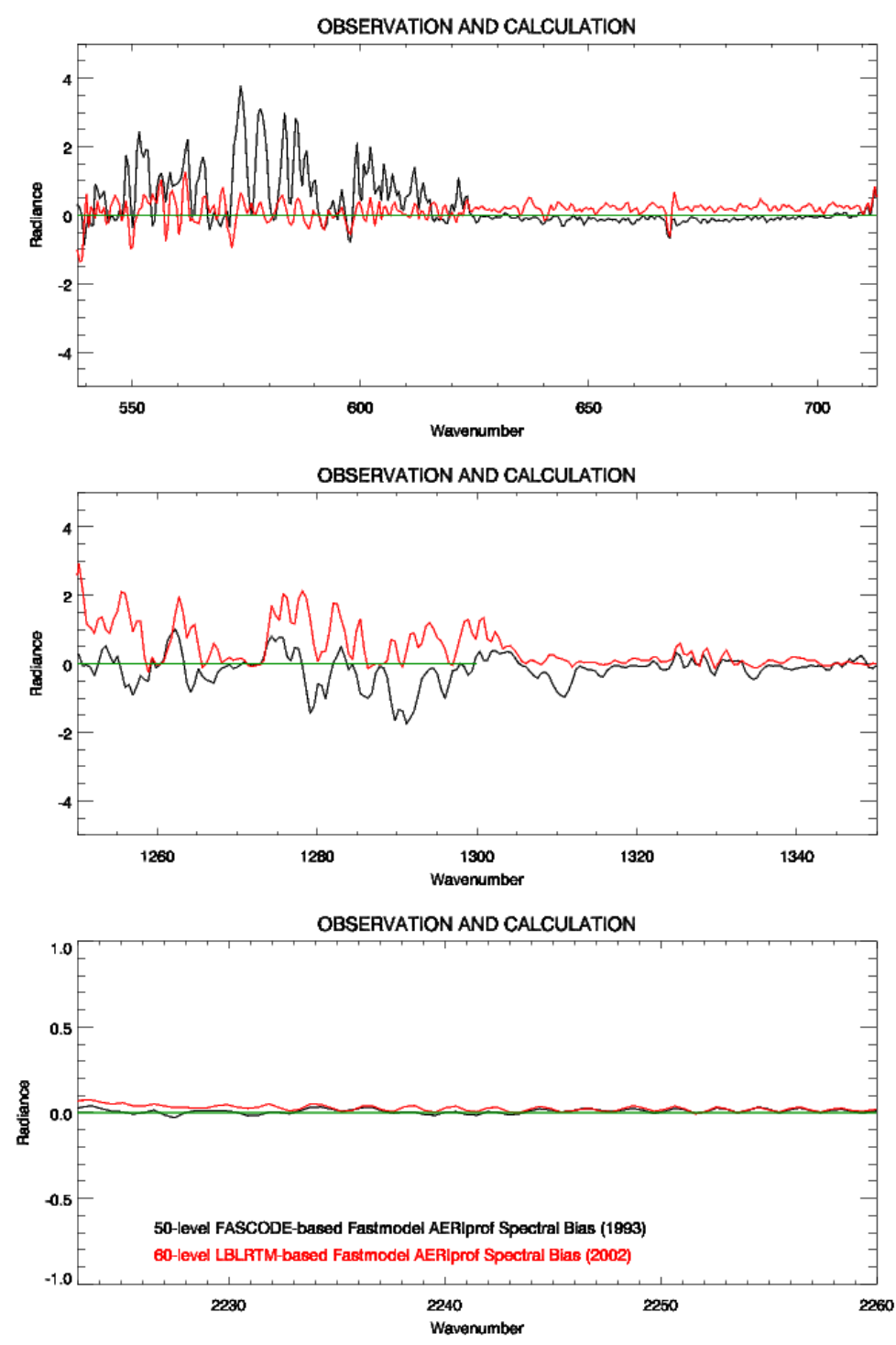

Figure 3: Mean spectral differences between observed AERI radiances and fast-model calculations using MWR-scaled radiosondes as input into the model. The red line differences are from calculations based on the current fastmodel (developed from LBLRTM 6.01 in 2002), while the black line differences are from the old fastmodel developed in 1993 using FASCODE. Differences are only shown in regions where the AERI statistical and physical temperature and moisture retrievals use radiance information. The top panel includes spectral regions used in both the $\mathrm{T}$ and $\mathrm{q}$ retrievals, while the spectral regions in the middle and lower panel are used only in the $q$ and $T$ retrievals, respectively.

The regressions used in the statistical retrieval were built using data from a large number of representative radiosonde profiles from the SGP region. Eigenvector analysis was used to relate the computed spectra from these radiosondes to the input atmospheric state profiles, allowing the atmospheric state to be retrieved statistically from the AERI's observed spectrum. Because the weighting functions for the 
spectral elements used in the retrieval peak are at or near the surface, the statistically retrieved profile converges to the mean atmospheric state above the boundary layer (around 2-3 km AGL). This statistical retrieval is site dependent. From July 1994-May 1996, more than 1000 clear-sky radiosondes from July 1994-May 1996 that covered a multitude of atmospheric conditions were used at the SGP sites to generate the statistical retrieval eigenvectors/regression coefficients. The 1Feltz version of AERIPROF used this statistical retrieval as its only source of first-guess information for the physical retrieval.

Because the information content in the AERI radiance diminishes with height, the retrieved profiles (for both the statistical and physical retrievals) are limited to altitudes below approximately $3 \mathrm{~km}$. However, if T/q profiles from other data sources are used as part of the first guess, more accurate profiles of T/q in the mid-troposphere can be realized. The 2Feltz version of AERIPROF (also called AERI-GOES) used the T/q profiles retrieved from the GOES radiance data in a 3x3-pixel (30x30-km region) around the AERI location as part of the first guess. The GOES profiles were blended with the statistical first guess that was described above for profiles taken between 2-3 km, and the statistical retrieval was used below 2 $\mathrm{km}$. The GOES T/q profiles were used above $3 \mathrm{~km}$, and a linear combination of the two in this altitude region. This merged profile was then used as the first-guess profile for the physical retrieval. This merged first guess took advantage of the sensitivity of the GOES radiance observations to temperature and water vapor in the upper and middle troposphere and the AERI's sensitivity to the lower troposphere, resulting in retrieved T/q profiles that spanned the entire troposphere in clear sky conditions. However, the GOES T/q retrievals are not performed in cloudy (e.g., cirrus) conditions, and, in these cases, the first guess used in the $2 \mathrm{Feltz}$ version consisted solely of information from the statistical retrieval.

To provide full tropospheric profiles of T/q for all conditions, an accurate source of middle and upper tropospheric data was needed that existed for all times and conditions. The Rapid Update Cycle (RUC; Benjamin et al. 1994, 1995) numerical weather prediction model fulfilled this requirement. The RUC model assimilates in-situ data from radiosondes and aircraft temperature reports, as well as remotely sensed data such as PWV and cloud top pressures from GOES and wind profiler data. As such, the middle and upper tropospheric profiles of T/q are reasonably accurate; however, the 20-km resolution of the model does not accurately portray the thermodynamic state of the boundary layer. For example, the nocturnal temperature inversion is often not well captured by the RUC model, and biases in the RUC moisture profile in the boundary layer frequently occur. However, the RUC T/q profiles are computed every hour regardless of clouds, thus the RUC output is an optimal source of middle and upper tropospheric information for the AERIPROF first guess. The 3Feltz version combines the RUC and statistical first-guess profiles in the same manner as the 2Feltz version combined the GOES data with the statistical first guess. The physical retrieval then modifies the merged first-guess profile in the boundary layer to match the observed AERI radiance data to provide an accurate T/q retrieved profile.

The AERIPROF algorithm has been extensively validated in clear skies in the SGP region. The water vapor profiles were compared to MWR-scaled radiosondes and Raman lidar data, showing agreement within 5\% in RMS in the water vapor mixing ratio profiles (Turner et al. 2000). The retrieved temperature profiles were also compared with coincident radiosonde observations showing RMS differences of less than 1,000 in the first $3 \mathrm{~km}$ (Feltz et al. 2003).

The fast-transmittance model uses a fixed pressure level grid for the vertical coordinate, and, thus, the retrieved profiles are in terms of pressure rather than height. However, the 1Feltz and 2Feltz versions of the algorithm used the hypsometric equation to interpolate the data to a fixed height grid. This introduces 
a small component of interpolation error into the retrieved profiles. Therefore, to preserve accuracy the retrieved profiles from the 3Feltz version of the algorithm are output on the original pressure grid. Because many of the ARM VAPs that use the AERIPROF data as input expect these profiles to be on a constant height grid, the retrieved profiles are also interpolated to a constant height grid. Therefore, the 3Feltz output files generated by ARM contain the same data on two different vertical grids.

Another improvement in the 3Feltz version of AERIPROF, relative to the earlier versions, is the T/q profile is provided for every AERI sample regardless of the success of the physical retrieval. This was only possible because the algorithm uses RUC data as part of the first-guess profile. If the retrieval is performed and converges successfully, then the output profile is the physically retrieved profile. However, if the retrieval is unsuccessful (e.g., clouds are too low, algorithm is unable to converge, or one of the quality checks indicates a problem with the physically retrieved profile), then the RUC profile is used as the output sample. A special field (named "profile_source_flag") indicates where the AERI data provided information in the output profile.

Figures 4 and 5 provide examples of 24-hour cross-sections of AERIPROF temperature and moisture retrievals, with Figure 5 magnifying the boundary layer on June 12, 2004. This case was selected because the illustration shows (a) low clouds, (b) mid-level clouds, (c) spikes in the AERI-retrieved temperature due to cloud contaminated radiance, and (d) a nighttime inversion. Cloud-base boundaries (as measured by the Vaisala ceilometer, one of the input datastreams used in AERIPROF) are indicated by the plus signs. These cross-sections show the diurnal cycle of nighttime inversion development between $0000-1200$ UTC and daytime boundary layer growth due to solar radiation input to the surface from $1200-2400$ UTC. The third panel within each of these figures shows where AERI radiance data (green area) are influencing the RUC profile background (orange area). This feature (allowing RUC-only profiles to be included in the output file when no AERI retrieval is possible) was included so continuous records of T/q are available to downstream VAPs that use the AERIPROF data. Notice that AERI retrievals are not available during times of extensive clouds (between 1500-1800 UTC). There are even some retrieval instabilities when clouds are not detected by the lidar but are still within the field of view of the AERI (seen as warm spikes in the retrieved temperature between 1800-2100 UTC on this day). These cloud-contaminated retrievals can be identified by comparing PWV computed from the AERIPROF retrieved q profile with the MWR's retrieved value, if the MWR's PWV is not used as a constraint in the AERIPROF retrieval (the ARM default is to not use MWR data in the retrieval for this reason). Figure 6 shows three comparisons of radiosondes and AERIPROF retrievals at 0530, 1130, and 2330 UTC on June 12, 2004. Notice, in general, temperature differences are less than $1 \mathrm{~K}$, while water vapor mixing ratio differences grew as the magnitude of the vertical gradient in the radiosonde profile increased. The 1130 UTC profile shows rapid transitions of water vapor gradient in the vertical; however, the infrared weighting functions are too broad at this altitude to resolve this structure. The AERI retrieval then represents a mean water vapor amount over a larger altitude depth than is resolved in the radiosonde profile. Notice the 2330 UTC mixing ratio structure has become well mixed by late afternoon, and the AERI retrieval represents this structure quite well due to the smooth vertical nature of the water vapor profile. 

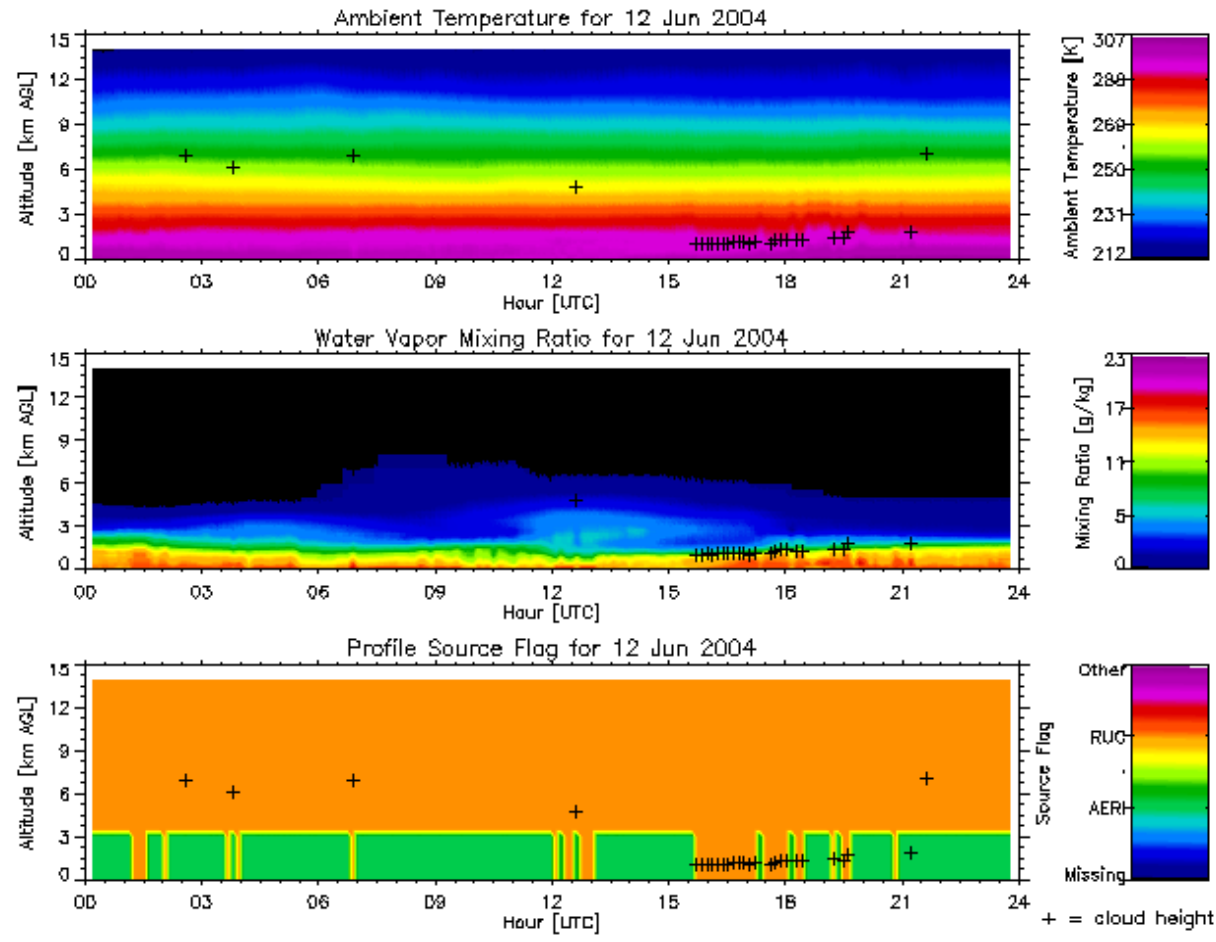

Figure 4: AERIPROF time-height temperature, moisture and data availability cross-sections indicating where RUC profiles and AERIPROF retrievals are available from the surface to 14 kilometers for June 12 , 2004, at the SGP Central Facility.
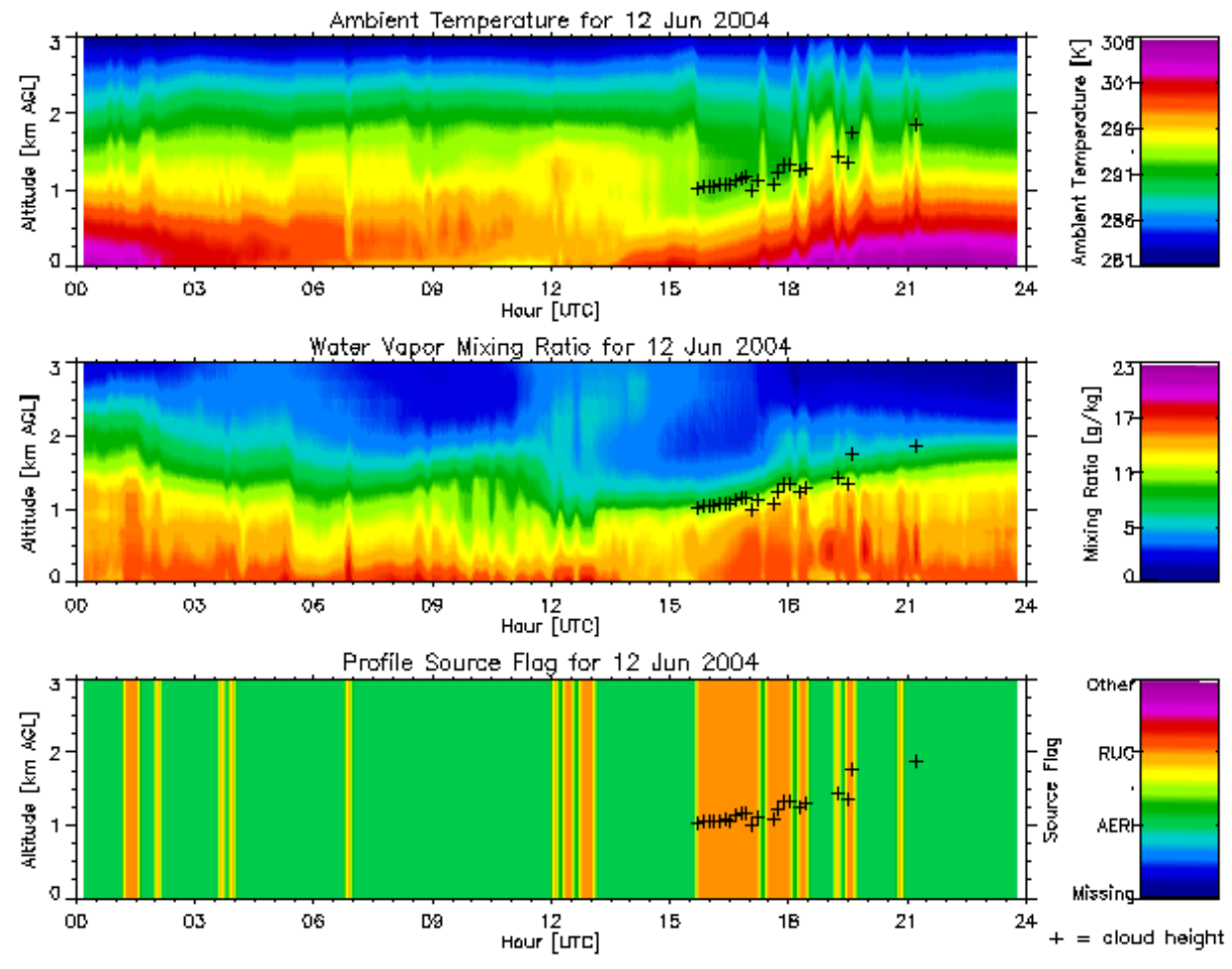

Figure 5: Same as Figure 4, but highlighting the lower troposphere. 

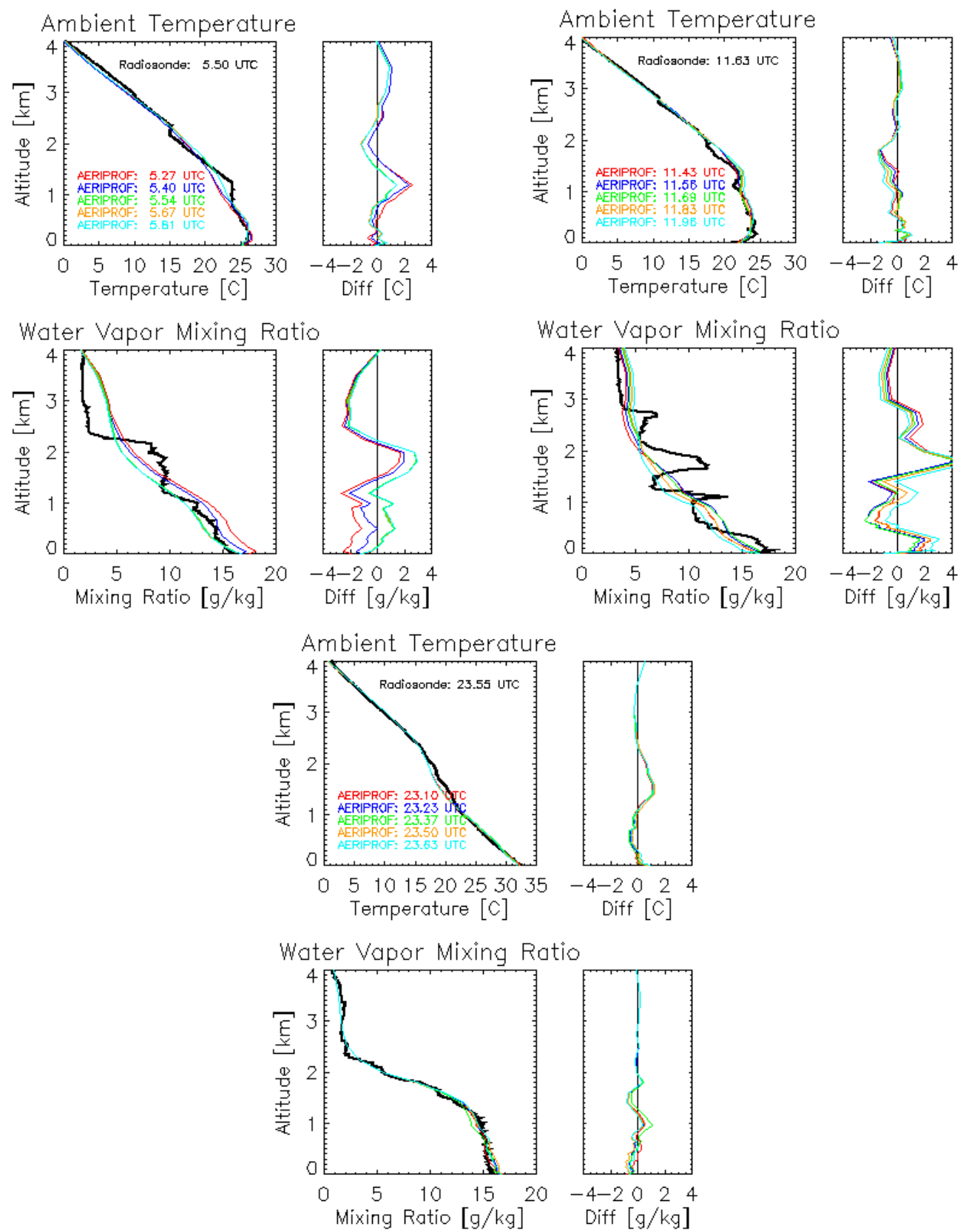

Figure 6: Several examples of AERIPROF temperature and water vapor retrievals compared to radiosonde launches on June 12, 2004.

\section{Algorithm Overview}

The AERIPROF algorithm is written primarily in Fortran77 and consists of more than 100 separate subroutines and functions. It runs on Unix workstations (both Solaris and Linux). Many of these functions, listed in the order they are called, are briefly described in Appendix B. The AERIPROF routine reads in ARM data directly from netCDF files and thus must be compiled with the netCDF library. Two input files are used to control the routine's execution, and several static binary coefficient files are read in by the routine. The binary coefficient files contain the fast-model regression coefficients, 
the regression coefficients used to generate the statistical first guess, and the bias and RMS spectra. The routine creates 3 output files for each run: a netCDF file containing the first-guess profiles used in the physical retrieval (named yyyymmddFG.cdf), a netCDF file containing the physically retrieved profiles with the RUC profiles inserted for samples where the retrieval did not converge (named yyyymmddAP.cdf), and an ascii log file. The algorithm processes one day at a time.

To execute the algorithm, the typical command line is

$$
\% \text { aeripl60 yyyymmdd } 1 \text { AERIPLUS.VIP }
$$

where the first argument is the date to process, the second argument turns on (1) or off (0) the ascii output to the screen, and the third argument is the name of the "variable input parameter (VIP)" file. The VIP file defines the paths needed by the algorithm to find the input data and the binary coefficient files. It also contains some of the basic toggle switches that can be used to preferentially select one data source over another (e.g., to use the cloud base height from the Vaisala ceilometer instead of from the micropulse lidar). An example of a VIP file used at CIMSS is given in Appendix C.

The other critical input ascii file that controls the execution of the algorithm is the "static input parameter (SIP)" file, which is expected to have the name AERIPLUS.SIP. (Note that the path to this file is defined in the VIP file.) The SIP file defines many of the thresholds and sets many of the options needed by the retrieval algorithm. For example, the SIP file example in Appendix D has a maximum cloud pressure allowed (CloudSearchMaximumPressure) set to $800 \mathrm{MB}$; therefore, if a cloud is located at a level of higher pressure (i.e., closer to the surface), then the retrieval is not performed. Care should be exercised when changing the SIP file parameters, because, in some cases, the quality of the retrieved profiles can be greatly affected. In general, the SIP file is only changed when it is desirable to run the algorithm in a special research mode.

Logic flow charts for AERIPROF are given in Appendix E. Flowchart 1 contains an overview of the retrieval logic. The retrieval is initiated with using the command line described above. The AERI radiances, surface data, NWP (RUC) profiles, and ceilometer data are read into the AERIPROF.f module where data quality control and time interpolation preprocessing is implemented. The data arrays are then passed into the core retrieval routine called aeriret.f. Several quality control tests are applied at the beginning of the aeriret.f module; these are described in more detail in following flowchart descriptions. Within aeriret.f, an optimal first guess is calculated and used within a physical retrieval. The retrieval is iterated to minimize the difference between the AERI radiance observation and forward calculation until a fixed residual threshold is met. If the threshold is not met or the residual begins to increase rather than decrease, then retrieval is aborted and flagged. A quality control flag is applied to each possible retrieval, and the resulting profiles and flags are written to netCDF output files containing the derived first-guess and physical retrievals. An ascii log file also contains information with regard to retrieval quality and provides a useful tracking mechanism for algorithm problems.

Flowchart 2 describes the global inputs and preparation logic for AERIPROF. The VIP file is read into AERIPROF, which defines input file paths/names and determines if files exist at all. The SIP file defines algorithm threshold and science parameters after which all auxiliary input files are read. After input files and global attributes are ingested into AERIPROF, each input data type is quality controlled and put into array structures. 
Preprocessing, quality control, and interpolation of auxiliary data to AERI radiance time steps are shown in flowchart 3. AERI radiances are ingested and apodized to reduce the impact of the instrument response function. AERI radiances are then converted to brightness temperature and quality control of the AERI spectrum is performed. This primarily involves checking for negative radiances and making sure the radiance is within a certain $\mathrm{min} / \mathrm{max}$ brightness temperature regime. If the quality control tests are not passed then this record is flagged and sent directly to the output netCDF file. When an observed radiance spectrum successfully passes the QC, the auxiliary datasets, including the hourly RUC2 analysis, are then interpolated to the AERI sample time.

The core routine (aeriret.f; Flowchart 4) contains the essential the retrieval calculation logic. After preprocessing, the appropriate spectral elements (regions defined within the SIP file) are extracted for use in the retrieval inversion methodology. Several spectral tests are performed to determine if the AERI radiance contains emission from clouds. If lidar data exists and the pressure at the cloud-base height is above the maximum pressure defined in the SIP file, then the retrieval is skipped with a QC flag indicator. If the cloud base is above this pressure level, then a spectrum with cloudy emission occurring at that level is computed and an "under cloud" retrieval is attempted within the aeriret.f routine. AERI radiances with no lidar data associated with them are checked for clouds by comparing the brightness temperatures between an opaque $\mathrm{CO}_{2}$ line and an infrared window spectral element. This difference must be greater than the threshold defined in the SIP file. If this test is not passed (which is a strong indication that a cloud exists within spectrum), the spectrum is flagged as cloudy with no cloud-base height determined and the retrieval is skipped. If the AERI radiance spectrum is determined to be clear or containing emission from a cloud higher than the maximum pressure level (where the base is defined by active lidar system), the spectrum is passed to the module getpro.f where the first guess is derived. The methodology within this subroutine is described in section IV above. The first guess is then passed back to aeriret.f and surface moisture (the source is specified by the SIP file) is inserted into the surface level of the profile along with a surface temperature derived from the $15 \mu \mathrm{m}$ region of the AERI radiance. This first guess is then smoothed (smoothing parameters defined in the SIP file) and this smoothed first guess will be written to the FG.cdf output file. The first guess is now used within the physical retrieval methodology (flowchart 5). The physical retrieval must pass a series of quality control checks and then is sent to the physical retrieval netcdf output file. If the retrieval does not pass QC, then the time-interpolated RUC profile is sent to the output file with a flag indicating that the profile is all RUC. After processing this record the loop continues on to the next record.

Flowchart 5 describes the physical retrieval procedure (described in detail by Smith et al. 1999). The first guess can be scaled to match the total precipitable water vapor value from microwave radiometer data. Currently this scaling is not performed within AERIPROF so that the MWR data can be used as an independent way to evaluate the AERIPROF retrievals. If the radiance was determined to contain clouds (via aeriret.f) the subroutine cloud2c.f computes a simulated cloudy spectrum using the first guess profile and cloud base height. Once this is done or the spectrum is clear, a total transmittance is calculated from the fast model (described in section III) from which radiance is computed in radcalc.f. The onion peel inversion methodology is accomplished with subroutines getrstr.f, ccam2.f, and recret.f. Adjustments to the former first guess profile are applied to create a new first guess profile. This profile is quality controlled and differences between the calculated radiance and observed radiance is integrated into a single RMS difference for all spectral elements used in the retrieval which must pass a convergence threshold set in the SIP file. If this threshold is not passed then the procedure in flowchart 5 is repeated. 
Also, if the RMS difference is not reduced through the iteration procedure then the physical retrieval is aborted and flagged as invalid. When the convergence criteria are met within a fixed number of iterations (defined in the SIP file) the retrieval is passed to the output file with proper flags ending the physical retrieval for this record.

\section{Quality Control}

Below is a description of quality control checks for both the input data for the AERIPROF retrieval algorithm and the retrieved AERI profiles.

\section{Input File Quality Control}

Surface data:

0: Surface data OK

1: Surface dewpoint calculated with AERI relative humidity due to bad surface station dewpoint $>2$ : Quality control flags within surface station meteorological data indicate that one of more of the surface temperature, pressure, or relative humidity is bad

Lidar data:

0: Lidar data $\mathrm{OK}$

1: Fog/condensation on lidar window

2: No data within AERI specified time period

3: No valid data found within LIDAR file

\section{Retrieval Profile Rejection Flags}

These flags are assigned to each AERI record number (radiance) that is processed through AERIPROF and are available in the AERIPROF physical retrieval netCDF file. Note that multiple error conditions might be possible for any given sample; however, the algorithm only reports the source of the first error condition encountered.

0: OK - Retrieval has passed all quality control checks.

1: Double saturation - Two levels in the first guess, iterated guess, or physically retrieved profile are at $100 \%$ saturation.

2: Sky conditions uncertain - Conflicting cloud information is occurring between the lidar and AERI spectra.

3: Low cloud - A cloud exists that is at an altitude below that corresponding to the maximum pressure level defined in the SIP file.

4: Cloud signal too large - If lidar data are unavailable and AERI radiances show brightness temperature differences between the most opaque $\mathrm{CO}_{2}$ region and window spectral region that is below the threshold set in the SIP file.

5: Residual too large - If the residual after maximum iterations is not below residual threshold set in SIP file or convergence fails before all iterations and residual not below threshold.

6: NULL input - AERI radiances do not exist in AERI radiance netCDF file.

7: Negative radiances - If negative radiances exist in AERI radiance after apodization.

8: Gross-error check failed - Radiance tests performed in aeriqc.f. 
9: Hatch-open test failed - AERI radiances show brightness temperature differences of less than the SIP-defined threshold between the most opaque $\mathrm{CO}_{2}$ region and window spectral regions 10: No RUC data - No upper-level temperature and moisture data available, retrieval is performed but only from surface to three kilometers using the regression retrieval as a first guess.

NOTE: Flags 1-9 indicate why the AERIPROF physically retrieved profile is not output. For these samples, the 3Feltz algorithm inserts the RUC profile into the output netCDF file.

\section{Implementation by the ARM Program}

To facilitate upgrades to the AERIPROF algorithm and to provide a common user interface to match the look and feel of the other VAPs, ARM developers have elected to use a controlling "wrapper" script to execute this VAP. This script, which is written in Perl, copies the needed input data from the ARM data tree into a working directory defined by the VIP file and renames them appropriately. The script then executes the AERIPROF executable that generates the three output files indicated above. The wrapper script then executes an "ingest" routine that reads in the physically retrieved profiles in the AP.cdf file and writes them into a netCDF file that follows ARM's data file standards. A set of quicklook images are then created, and the working directory is cleaned up. The data that was output into the first-guess netCDF FG.cdf file are not kept.

\section{Input Data}

The AERIPROF algorithm requires AERI radiance data from both channel 1 and channel 2 (500-1800 and $1800-3000 \mathrm{~cm}^{-1}$, respectively) and surface pressure and humidity data. However, more accurate retrievals can be realized if information is available on the temperature and water vapor profile in the middle and upper troposphere (from the RUC model, the GOES retrievals, or interpolated radiosondes, in order of preference). Also, if the retrieval is performed in cloudy conditions, a measurement of the cloudbase height from the Vaisala ceilometer, the micropulse lidar, or the Belfort laser ceilometer greatly improves the algorithm's ability to discriminate the cloud signal from the T/q signal. Finally, an estimate of the PWV from the MWR greatly improves the statistical first guess; however, not using the MWR data in the retrieval allows it to be used by the investigator as an independent source of quality control.

An additional input, which has only been used in research mode, is the water vapor profile observed by the Raman lidar. Because there is absorption by water vapor in the $\mathrm{CO}_{2}$ bands used for the temperature retrieval, inputting an accurate water vapor profile will significantly improve the accuracy of the retrieved temperature profile. If Raman lidar data are found in the directory specified by the VIP file, these data will be used for the water vapor profile, and the retrieval algorithm will only retrieve the temperature profile. However, because the Raman lidar data are not available away from the SGP Central Facility, this option is not used operationally in ARM.

The table below summarizes the input data and their criticality for the AERIPROF algorithm.

Required:

sgpaeri01ch1C1.\{a1,b1\}

sgpaeri01ch2C1.\{a1,b1\}

sgp1smosE13.a0 or sgpthwapsC1.a1 or sgpokmesoxxxx 
Highly recommended:

sgpruc20isobX1.c1 and sgpvceilC1.\{a1,b1\} or sgpmplnorC1.c1 or sgpblcC1.a1

Optional:

sgpmwrlosC1.\{a1,b1\} recommended, unless desired to use for independent QC

Research mode:

sgp10rlprofmr1turnC1.c1

\section{Output Data}

As mentioned above, the AERIPROF algorithm runs inside a wrapper script and not all of the output files generated by the AERIPROF binary are written into the ARM data tree. The output files created by the AERIPROF algorithm include the following:

$\begin{array}{ll}\text { yyyymmdd.log } & \text { - ascii log file that is not kept } \\ \text { yyyymmddFG.cdf } & \text { - netCDF file with first-guest profile that is not kept } \\ \text { yyyymmddAP.cdf } & \text { - ingested as sgpAERIPROF3 feltzC1.c1 }\end{array}$

The AERIPROF3FELTZ algorithm produces two daily netCDF files. The complete netCDF file contains all data quality information; the summary file contains a condensed summary of the data quality information. The VAP also produces daily quicklooks.

The daily AERIPROF3FELTZ VAP output files follow the ARM naming convention [site]aeriprof3feltz[facility].LL.YYYYMMDD.hhmmss.cdf

where

[site] is the ARM site name (SGP, NSA, TWP);

[facility] is the facility name $(\mathrm{C} 1, \mathrm{C} 2, \mathrm{E} 1, \mathrm{E} 2, \mathrm{etc})$;

LL is the level of the datastream;

$\mathrm{LL}=\mathrm{c} 1$ for the netCDF file with the bit-packed QC information and LL $=\mathrm{s} 1$ for the summary file;

YYYY is the year;

$\mathrm{MM}$ is the month of the year;

$\mathrm{DD}$ is the day of the month;

hh is the hour of the day of data start;

$\mathrm{mm}$ is the minutes of the hour;

and ss is the seconds of the minute.

\section{AERIPROF Retrieval Notes of Interest:}

Listed below are issues which AERIPROF users should be aware of when executing the AERIPROF code. The issues are caveats and notes of interest that may help users understand retrieval profile irregularities.

a. For optimal retrieval results, surface moisture and a best guess of atmospheric state from an NWP model (i.e., RUC2) should be provided at a minimum as input to AERIPROF. 
b. During climatologically dry periods high thin clouds (greater than $5 \mathrm{~km}$ ) may not be detected by the Vaisala lidar and the AERI radiances may be declared clear because of the lack of significant impact on the atmospheric microwindows within the spectra. This may cause the AERI water vapor profile to moisten due to the contribution of the cirrus emission within the water vapor retrieval regions. Futher work needs to be done to provide QC during these meteorological regimes.

c. A quality control test (that has yet to be implemented in AERIPROF) to remedy issue (b) is to compare the integrated water vapor from AERI to the microwave radiometer. The water vapor amounts should increase significantly during periods of cloud contamination within the AERI radiances.

d. The statistical regression retrieval for AERIPROF is SGP site specific. New regression datasets need to be developed for the other ARM sites to apply this retrieval methodology. Also, highquality NWP model profiles would be needed for the NSA, TWP, Darwin, and for the specific locales where the ARM Mobile Facility is deployed.

\section{References}

Benjamin, SG, KJ Brundage, and LL Morone. 1994. "The rapid update cycle. Part I: Analysis/model description. Technical Procedures Bulletin,” No. 416, NOAA/NWS, 16 pp. [National Weather Service, Office of Meteorology, 1325 East-West Highway, Silver Spring, MD 20910].

Benjamin, SG, D Kim, and TW Schlatter. 1995. "The rapid update cycle: A new mesoscale assimilation system in hybrid theta-sigma coordinates at the National Meteorological Center." Preprints. Second Int. Symp. On Assimilation of Observations in Meteorology and Oceanography, Tokyo, Japan, Japanese Meteorological Agency, 337-342.

Clough, SA, FX Kneizys, LS Rothman, and WO Gallery. 1981. "Atmospheric spectra transmittance and radiance: FASCODE1B.” SPIE Atmos. Transmission 277:152.

Clough, SA, FX. Kneizys, and RW Davies. 1989. "Line shape and the water vapor continuum." Atmospheric Research 23:229-241.

Clough, SA, MJ Iacono, and JL Moncet. 1992. "Line-by-line calculations of atmospheric fluxes and cooling rates: Application to water vapor." Journal of Geophysical Research 97:15761-15785.

Clough, SA, and MJ Iacono. 1995. "Line-by-line calculations of atmospheric fluxes and cooling rates II: Application to carbon dioxide, ozone, methane, nitrous oxide, and the halocarbons." Journal of Geophysical Research 100: 16,519-16,535.

Eyre, J.R. 1991. "A fast radiative transfer model for satellite sounding systems." ECMWF Research Department Technical Memorandum 176. 
Feltz, WF. 1994. Meteorological applications of the atmospheric emitted radiance interferometer (AERI). M. S. thesis, Department of Atmospheric and Oceanic Sciences, University of WisconsinMadison, 87 pp. [Available from University of Wisconsin-Madison, Schwerdtfeger Library, 1225 W. Dayton, Madison, Wisconsin 53706].

Feltz, WF, WL Smith, RO Knuteson, HE Revercomb, HM Woolf, and HB Howell. 1998. "Meteorological applications of temperature and water vapor retrievals from the ground-based atmospheric emitted radiance interferometer (AERI)." Journal of Applied Meteorology 37:857-875.

Feltz, WF and JR Mecikalski. 2002. "Monitoring High Temporal Resolution Convective Stability Indices Using the Ground-based Atmospheric Emitted Radiance Interferometer (AERI) During the 3 May 1999 Oklahoma/Kansas Tornado Outbreak.” Weather Forecasting 17:445-455.

Feltz, WF, D Posselt, JR Mecikalski, GS Wade, and TJ Schmit. 2003. "Rapid boundary layer water vapor transitions." Bulletin of the American Meteorological Society 84:29-30.

Feltz, WF, HB Howell, RO Knuteson, HM Woolf, and HE Revercomb. 2003. "Near continuous profiling of temperature, moisture, and atmospheric stability using the atmospheric emitted radiance interferometer (AERI)." Journal of Applied Meteorology 42:584-597.

Garand, L, DS Turner, M Larocque, J Bates, S Boukabara, P Brunel, F Chevallier, G Deblonde, R Engelen, M Hollingshead, D Jackson, G Jedlovec, J Joiner, T Kleespies, DS McKague, L McMillin, J-L Moncet, JR Pardo, PJ Rayer, E Salathe, R Saunders, NA Scott, P Van Delst, and H Woolf. 2001. "Radiance and Jacobian intercomparison of radiative transfer models applied to HIRS and AMSU channels." Journal of Geophysical Research 106:24 017-24 031.

Revercomb, HE, DD Turner, DC Tobin, RO Knuteson, WF Feltz, J Barnard, J Bösenburg, D Cook, R Ferrare, J Goldsmith, S Gutman, R Halthore, B Lesht, J Liljegren, H Linné, S Melfi, J Michalsky, V Morris, W Porch, S Richardson, B Schmid, M Splitt, T Van Hove, E Westwater, and D Whiteman. 2003. "The Atmospheric Radiation Measurement (ARM) Program's Water Vapor Intensive Operational Periods: Overview, accomplishments, and future challenges." Bulletin of American Meteorological Society 84:217-236.

Rodgers, CD. Inverse methods for atmospheric sounding: Theory and practice. World Scientific Publishing Co. Ltd., 2000.

Schmit, TJ, WF Feltz, WP Menzel, J Jung, JP Nelson III, and GS Wade. 2002. "Validation and use of GOES Sounder Moisture Information.” Wea. Forecasting, 17, 139-154.

Smith, WL, WF Feltz, RO Knuteson, HE Revercomb, HB Howell, and HM Woolf. 1999. "The retrieval of planetary boundary layer structure using ground-based infrared spectral radiance measurements." Journal of Atmospheric and Oceanic Technology 16:323-333.

Turner, DD, WF Feltz, and RA Ferrare. 2000 "Continuous water profiles from operational ground-based active and passive remote sensors." Bulletin of the American Meteorological Society 81:1301-1317. 
Turner, DD, BM Lesht, SA Clough, JC Liljegren, HE Revercomb and DC Tobin. 2003. "Dry bias and variability in Vaisala RS80-H radiosondes: The ARM experience." Journal of Atmospheric and Oceanic Technology 20:117-132.

Turner, DD, DC Tobin, SA Clough, PD Brown, RG Ellingson, EJ Mlawer, RO Knuteson, HE Revercomb, TR Shippert, and WL Smith. 2004. "The QME AERI LBLRTM: A closure experiment for downwelling high spectral resolution infrared radiance." Journal of Atmospheric Science 61:2657-2675. 


\section{Appendix A: AERIPROF Heritage}

- 1989-1991 - Retrieval algorithm was developed based on Bomem MB100 single detector radiances from the "Baby HIS" instrument. This algorithm was developed using 386/486 PC technology with Watcom Fortran in an OS/2 environment. Primary work was done by Bill Smith and Ben Howell.

- 1991-1992 - Algorithm was successfully compiled on an AIX IBM Workstation, which allowed faster retrieval calculation. A two-channel retrieval algorithm was developed to accommodate the extra radiance information the newly acquired MB120 provided. Algorithm successfully performed retrievals on SPECTRE, STORMFEST, and CAMEX I retrievals. Smoothed radiosonde was used as a first guess of atmospheric state for the algorithm.

- 1993-1994 - ARM funding provided the resources necessary to improve the algorithm to an operational state. Statistical retrievals based on 600 clear radiosondes were implemented into the algorithm instead of using smoothed radiosonde data for the first guess profile. This provided a means to automate the algorithm and allow it to be truly operational. A new fast model was added with updated transmittance coefficients based upon HITRAN 1993. Error was found in water vapor retrieval portion of algorithm and was corrected.

- 1995 - A full rewriting of the retrieval software modularized the retrieval code. This was done to allow easy updates to different parts of the algorithm and to provide Fortran subroutines for which a $\mathrm{C}$ wrapper could be placed around. Ancillary data was allowed to help the first guess of atmospheric state and to provide information about cloud altitude. Surface meteorological, total precipitable water, and cloud altitude data were added as optional data inputs to augment the first-guess profile and further constrain the retrieval process. This ARM operational retrieval algorithm (AERIPROF version 1) was implemented in June 1995.

- 1996 - The retrieval algorithm was modified to allow superadiabatic lapse rates in the first 100 meters of atmosphere. New statistical retrieval coefficients were added which were based upon 1200 clear radiosondes from the ACRF SGP site. A new spectral bias was calculated from microwave radiometer PWV scaled radiosondes launched during the 1996 Water Vapor IOP.

- 1997 - The 40-level retrieval algorithm was corrected to the 50 fast-model levels to eliminate the interpolation error that was required for level adjustment. AERI software papers were written for peer-reviewed journals.

- 1998 - The AERI retrieval software has been modified to allow GOES physical retrievals and LIDAR water vapor profiles to be used in retrieval processing. This became version 2 of AERIPROF and was implemented in the ARM data system in early 1999.

- 2000 - RUC hourly analysis profiles collocated over SGP AERI systems are implemented into the first guess instead of GOES sounder profiles alleviating missing GOES profile issues when high, thick clouds are present.

- 2002 - AERI fast model is updated with a 60-level model based on LBLRTM 6.0 and HITRAN 2000 which became version 3 of AERIPROF.

\section{Uses for ARM}

- Preliminary studies have been conducted to drive SCM/CRM calculations with AERIPROF retrievals and collocated wind profiler data including mass flux water vapor divergence and advection (Xie and Cederwall 2000). A grid of 5 AERI systems within the SGP site domain were deployed for a 5-year 
period (December 1998-December 2003) and collocated with operational wind profilers. Preliminary studies have been initiated to test SCM runs with remotely sensed temperature, moisture, and winds.

- AERIPROF retrievals using the NSA and Surface Heat Energy Budget of the Arctic (SHEBA) Extended Range AERI systems have been performed to provide input for Large Eddy Simulation (LES) parameterizations. Several NSA and SHEBA ER-AERI-ER data sets have been processed to validate and develop boundary layer Large Eddy Simulation (LES) models (in collaboration with Dr. J. Curry University of Colorado and Dr. K. Stamnes University of Alaska Fairbanks) in the Arctic climate.

- AERIPROF retrievals using the TWP AERI and Marine AERI instruments have been performed for Nauru 99. Preliminary Nauru Island TWP AERI retrievals have been calculated and compared to ship-based M-AERI retrievals. The research results indicate a Nauru heat island effect of 1.5 degrees Celsius (in collaboration with Dr. P. Minnett University of Miami and Dr. Ed Westwater NOAA ETL). Similar heat island effect temperature values have been determined with RASS (W. Brown, NOAA ATD).

- AERIPROF retrievals have been incorporated in best estimate of atmospheric state value-added products that combine Raman Lidar water vapor and AERIPROF temperature to provide 10- minute resolution best estimate thermodynamic state and aerosol profiles over the ACRF SGP Central Facility (Turner et al. 2002).

- AERIPROF retrieval applications have been implemented including objective detection of boundarylayer height and turbulent moisture plumes. A combined Raman Lidar/AERI algorithm is being developed to provide a consensus PBL height determination (in collaboration with Dr. Rich Ferrare of NASA/LaRC) for the SGP Central Facility at Lamont, Oklahoma. AERI temperature and moisture retrievals should provide a new source of data for LES studies.

- ARM-funded AERI retrieval improvement has also benefited other research programs such as validation of AIRS spectroscopy and atmosphere state (in conjunction with Dr. David Tobin SSEC UW-Madison). NOAA and NASA Satellite validation funding has provided additional resources to use the best available ARM datasets to reduce satellite derived product uncertainties (Hawkinson et al. 2003; Schmit et al. 2002; Tobin et al. 2003). 


\section{Appendix B: AERIPROF Subroutine Descriptions}

Aeripl60.f [main routine]*

Getvip.f [read in the variable input parameters]

Chksdat.f [logic to determine source of the input surface meteorology data]

Chkcdat.f [logic to determine source of cloud boundary information]

Fnames.f [logic to build pathnames to datafiles using date from command line and VIP info]

Getsip.f [logic to read static input parameters]

Ingcbh.f [reads cloud boundary netCDF file, returning arrays of cloud base height and time]

Ingsfc.f [reads in surface meteorology data, returning arrays of pres, rh, temp, and time]

Ingmwd.f [reads in total precipitable water vapor amount from MWR (not needed)]

Ingrwv.f [reads in profiles of water vapor mixing ratio from Raman lidar (not needed) $]^{\dagger}$

Inggoes.f [reads in the GOES retrieved water vapor profiles for first guess (not needed)]

Ingruc2.f [reads in the RUC2 model profiles for first guess]

Rdbcof.f [reads in the BIAS, RMS, and first-guess regression coefficient files]

Aerinfo.f [reads in the needed time-series data from the AERI data files]

Cnvrtau.f [converts the time-series data read in from the AERI into proper units]

Getmodl.f [gets the appropriate model atmosphere for this lat/lon/date - for trace gases]

Logic to create filenames for the output files

Creation of flags to indicate which data from what sources are being used in the retrieval

Loop over AERI radiance records

Get2bs.f [gets the radiance spectrum]

Apodiz.f [apply apodization]

Getabt.f [compute brightness temperature spectrum, capturing also local radiative air temp]

Aeriqc.f [quality control AERI radiance spectrum; no retrieval performed for bad data]

Getsfc.f [interpolate surface meteorological data to AERI sample time]

Gettpw.f [interpolate microwave PWV data to AERI sample time, if PWV available]

Getcbh.f ["interpolate" cloud base height data to AERI sample time, if data available]

Getrwv.f [interpolate Raman lidar data to AERI sample time, if data available]

Getgrp.f [interpolate GOES retrieval data to AERI sample time, if data available]

Getruc2.f [interpolate RUC2 data to AERI sample time, if data available]

Capture information on which data was available for this particular AERI sample time

Aeriret.f [CORE ROUTINE: Retrieve $\mathrm{q}(\mathrm{z})$ and $\mathrm{T}(\mathrm{z})$ profiles from AERI spectrum]

MORE DETAILS HERE ON NEXT PAGE

Gethts.f [compute the heights of the first-guess data using hypsometric equation]

Outret.f [output first-guess profiles to the output data file]

Gethts.f [compute the heights of the physically-retrieved data using hypsometric equation]

Outret.f [output physically-retrieved profiles to the output data file]

Outces.f [output cloud emissivity spectrum, if desired (set in SIP file)]

Close netCDF files for physically retrieved output

Close netCDF files for first-guess profiles, if needed

Close netCDF files for cloud-emissivity spectra, if needed

* This is done in an experimental mode. Supplying the water vapor profiles from the Raman lidar allows the AERI retrieval algorithm to retrieve a much more accurate temperature profile. Contact Wayne for details.

${ }^{\dagger}$ NOTE: Many variables are defined and described in aeripl60.f. 


\section{Aeriret.f [Core routine for AERI T/q retrievals]*}

Logic to determine which spectral elements to use for retrievals and cloud emissivity spectrum Comparison of $\mathrm{CBH}$ determined from AERI (in aeriqc.f) with lidar $\mathrm{CBH}$ [4 diff checks applied] Getpro.f [get profiles from radiance observation using statistical retrieval logic] ${ }^{\dagger}$

Logic to insert surface meteorological obs into the statistically retrieved profile If cloud exists, compute cloud-pressure level, and find closest pressure level used in retrieval Smooth the first-guess profile

Save the first-guess profile, if desired

Loop to perform physical retrieval

Blend in surface meteorology again (creating the "previous-guess" profiles)

Scale water vapor mixing ratio profile to agree with the PWV observed by the microwave

If clouds are present, compute cloud properties via cloud2c.f; perform some QC on cloud signals

Calculate total transmittance from previous-guess profile via ctpf $2 . f$

Compute radiance from transmittance profiles via radcalc.f

Compute Rstar spectrum (see Smith et al. 1999) with getrstr.f

Compute A matrix (??) with ccam2.f

Compute adjustments to the previous-guess profiles using onion-peel technique using recret.f

Apply adjustments to the previous-guess profile to create updated-guess profile

Apply some quality control (such as double-saturation checks, etc) to updated-guess profile

Compute RMS statistics to test for convergence

If retrieval is rejected (i.e., from quality control checks) default to RUC2 profile

Else move on to next AERI sample

* Can read in any first-guess profile here (like a radiosonde) if data are in "BRF" format and the flag ISRCFG $=1$

$\dagger$ NOTE: Many variables are defined and described in aeriret.f. 


\section{Appendix C: Variable Input File}

The Variable Input File (VIP) is one of two text files needed to execute the AERIPROF algorithm. When the VIP file is not present, a file called "SAMPLE.VIP" will automatically be derived, and the user must then fill in the proper paths where input files are located. A line-by-line explanation is provided below each italicized VIP line.

\section{AERIPLUS.VIP}

(Variable input parameters for AERIPROF)

NOTE: Blank lines and lines with "(" in col.1, are ignored.)

VIP file text descriptions

PathLogFile: = '/home/waynef/retrievals/realtime/lamont/'

Log file directory path

PathCoefficientFiles: = '/home/waynef/retrievals/coeffs/transmit/'

AERIPROF fast model transmittance coefficient files path

PathInputRadianceFiles: = '/home/waynef/retrievals/realtime/data/lamont/aeri/'

AERI radiance input file path

FirstInputRecord: $=1$

AERI radiance record number start

LastInputRecord: $=999$

AERI radiance record number end

PathOutputFile: = '/home/waynef/retrievals/realtime/lamont/data/'

netCDF output file path

PathCloudEmisFile:= '/home/waynef/retrievals/realtime/lamont/data/'

Cloud emissivity file path (if turned on in SIP file)

PathSurfaceDataFile: = '/home/waynef/retrievals/realtime/data/lamont/thw/'

Surface meteorological input data path

PathCloudBaseHeightFile: = '/home/waynef/retrievals/realtime/data/lamont/vceil/'

Lidar cloud base height input data path

PathMicrowaveRadiometerFile: = '/home/waynef/work/deliver/aeripl60/data/'

Microwave radiometer input data path

NameEignVectEignValuFile: ='/home/waynef/retrievals/coeffs/firstguess/eignvecs.001'

Eigenvector/Eigenvalue path and name for regression retrieval

NameEignVectCoefFile: = '/home/waynef/retrievals/coeffs/firstguess/eigncoef.001'

Eigenvector coefficient files path and name

NameMeanProfilesFile: = '/home/waynef/retrievals/coeffs/firstguess/meanprof.001'

Mean profile path and name for regression retrieval

NameEignVectTPWcoefFile: = '/home/waynef/retrievals/coeffs/firstguess/eigncoefpw.001'

Eigenvector coefficient path and file if microwave radiometer total PWV available

NameAERIrmsFile: = '/home/waynef/retrievals/realtime/lamont/AERIRMS.2B'

AERI radiance root mean square path and file

NameAERIbiasFile: = '/home/waynef/retrievals/realtime/lamont/AERIBIAS.2B'

AERI spectrum bias path and file 
SourceFlagCloudBaseData(0:MPL, 1:BFCL, 2:VCL):= 2

Parameter defining type of lidar data used for cloud-base data

PathStaticParameterFile: = '/home/waynef/retrievals/realtime/lamont/'

Path to SIP file

PathRamanWaterVaporFile: = '/home3/waynef/'

Path to Raman lidar data if used to determine water vapor (not used for ARM purposes)

PathGoesRetrievalFile: = '/home/waynef/work/deliver/aeripl60/'

Path to GOES retrieval file (RUC now used instead)

MaximumGOESTimeDiff(minutes): $=900.0$

Maximum GOES time difference

SourceFlagSurfaceData(0:SMOS, 1:THWAPS, 2:OKM):=0

Parameter defining type of ARM surface data

OKMesonetStationID: = 'PUTN'

If OKM set in line above, Mesonet name identifier

FirstGuessFile:= '/home5/waynef/retrievals/sonde_firstguess/sgpcart_sondes_nov99.brf'

If RUC or GOES not available, file of radiosondes can be used as first guess

PathRUC2file:= '/home/waynef/retrievals/realtime/data/lamont/ruc2/'

Path to RUC analysis profiles 


\section{Appendix D: Static Input Parameter}

The lines below describe the Static Input Parameter (SIP) file, which is the other text file necessary to run AERIPROF. This file defines parameter that should be held STATIC unless using the algorithm in a research mode. A line-by-line explanation is provided below each italicized SIP line.

\section{AERIPLUS.SIP}

NOTE: Enclose all character strings in single quotes!

(lines beginning with "(" are ignored)

SIP file text descriptions

ConstituentType(CTYPE):= 'ALL'

Paramater defining that "ALL" constituent types are used in fast model

EmissivityMinimum(EMIN): $=0.002$

Emissivity minimum in emissivity output (not used for ARM)

CoefficientPrefix(CFP): = 'AS'

Transmittance coefficient prefix used with coefficient names

SmootherPassCount(NPASS, $0=$ NO smoothing): $=100$

Amount of smoothing passes used on the first guess profile (flowchart 4)

SmootherLoAltPressure(PLO): $=1000$

Lowest altitude in millibars that smoothing is applied to first guess

SmootherHiAltPressure $($ PHI): $=600$

Highest altitude in millibars that smoothing is applied to first guess

SmootherWeight(SWT):= 1

Magnitude of smoothing

MaximumIterationNumber(MITER, $0=1$ pass only): $=5$

Maximum number of iterations defined for physical retrieval loop (flowchart 5)

PhysRetBiasSwitch $(Y / N):=$ 'W'

Switch defining part of spectrum AERIBIAS.2B should be applied: W- water vapor only, $\mathrm{Y}$ - all regions, $\mathrm{N}-$ no regions

GuessProfileShiftSwitch(Y/N): = 'N'

Switch determining if first-guess profile should be shifted to $\mathrm{Y}$ - fast model levels or $\mathrm{N}$ - levels normalized to the surface pressure

RadiativeSurfaceTemp $(Y / N):=$ ' $Y$ '

Switch to determine if the surface temperature applied to the AERI first guess should be determined from the spectrum $(\mathrm{Y})$ or surface data input $(\mathrm{N})$

TempSpectralRegion(1): = 612618

Definition of first temperature retrieval spectral domain to use from observed AERI radiances

TempSpectralRegion(2): $=624660$

Definition of second temperature retrieval spectral domain to use from observed AERI radiances

TempSpectralRegion(3): = 674713

Definition of third temperature retrieval spectral domain to use from observed AERI radiances

TempSpectralRegion(4): = 22232260

Definition of fourth temperature retrieval spectral domain to use from observed AERI radiances WaterVaporSpectralRegion(1): = 538588 
Definition of first water vapor retrieval spectral domain to use from observed AERI radiances WaterVaporSpectralRegion(2): = 12501350

Definition of second water vapor retrieval spectral domain to use from observed AERI radiances

RetrievalTopPressure (PTOP): $=700$

Upper-level profile constraint to physical retrieval iteration

RetrievalConditioningParameter(GAMMA): $=.001$

Conditioning parameter applied to inversion matrix for mathematical stability

RetrievalRestrictionCriterion(CRIT0): $=100$

Parameter needed to stabilize AERI retrieval inversion in recret.f

CloudSearchMinimumPressure $($ PCLD): $=500$

Upper-level atmospheric constraint on cloud search using AERI-only radiance data

CloudSearchMinimumAmount $(A C L D):=0.001$

Constraint on minimum cloud amount when determining if a cloud exists using radiances

CloudSearchMaximumPressure $($ PCMIN): $=800$

Low-level atmospheric constraint on cloud search using AERI only radiance data

CloudSearchMaximumAmount(AMTMX): = 0.4

Constraint on maximum cloud amount when determining if a cloud exists using radiances

CloudComputationSwitch $(Y / N):=$ ' $Y$ '

Switch to compute cloud altitude and amount using only AERI radiances

OutputCloudEmissivity $(Y / N):=$ ' $N$ '

Switch to compute spectrum of cloud emissivity

StackHeight(meters): $=0.0$

Meters defined to account for AERI chimney effect during first AERI deployment in Lamont trailer

OutputRSTAR(Y/N): = 'N'

Switch to output a binary file of spectral residual for each physical retrieval iteration

OutputWtFnSpectra $(Y / N):=$ 'N'

Switch to output a binary file of weighting functions for each physical retrieval

OutputInterimRetrievals: $=$ ' $N$ '

Switch to output each profile for each physical retrieval iteration until convergence is achieved

OutputFirstGuessProfiles: $=$ ' $Y$ '

Switch to output first guess profiles to netCDF file

StatRetBiasSwitch $(Y / N):=$ ' $Y$ '

Switch to apply the AERIBIAS.2B spectral residuals to AERI observation before regression is performed OutputStatRetProfiles: = ' $Y$ '

Switch to output statistical retrieval profiles

SurfacePressureLoLimit: $=900.0$

Constraint on minimum surface pressure allowed

SurfacePressureHiLimit: $=1020.0$

Constraint on maximum surface pressure allowed

SurfacePressureNominal: $=1000.0$

Definition of nominal surface pressure for profile normalization (not usually used)

OutputScreenMessages $(Y / N):=$ ' $Y$ '

Switch to output AERIPROF retrieval text to screen

OutputLogFileMessages $(Y / N):=$ ' $Y$ '

Switch to output algorithm messages to a log file

AERIsceneViewDuration(SVDUR):= 195.03 
Number of seconds the AERI is in sky-view mode; used to appropriately determine cloud base from lidar data MinTempDiffHatchOpen(TDHTCH): $=6.0$

Minimum temperature difference between opaque and IR window temperature used to determine if hatch was closed on AERI

MinTempDiffClearSky(TDVRFY): $=40.0$

Minimum temperature difference between opaque and IR window temperature used to determine if clouds are within spectrum MaxResidualBrTemp(RTSQLMX):= 0.8

Maximum residual brightness temperature threshold for determining if physical retrieval has met radiance convergence tempBlendHeightBottom(GTBHBTM, $\mathrm{km}):=0.8$

Bottom height used for blending RUC- and AERI-derived temperature profiles.

tempBlendHeightTop(GTBHTOP, $\mathrm{km}):=1.3$

Top height used for blending RUC- and AERI-derived temperature profiles dewptBlendHeightBottom(GDBHBTM, $\mathrm{km}$ ): = 1.0

Bottom height used for blending RUC- and AERI-derived dew point profiles

dewptBlendHeightTop (GDBHBTM, $\mathrm{km}):=2.0$

Top height used for blending RUC- and AERI-derived dew point profiles OutputOriginalRetrievals $(Y / N):=$ ' ${ }^{\prime}$

Flag for output of original retrieved temperature and pressure profiles

FirstGuessSource(0:ST.REG., 1:BRF):=0

Flag to use the statistical regression for the lowest layers of the first guess.

OutputRUCprofiles(1/0): $=1$

Flag to use the RUC profiles when AERI retrieval is not possible 


\section{Appendix E: AERIPROF Algorithm Flowcharts}

AERI retrieval software flow charts - 2004

1. overview

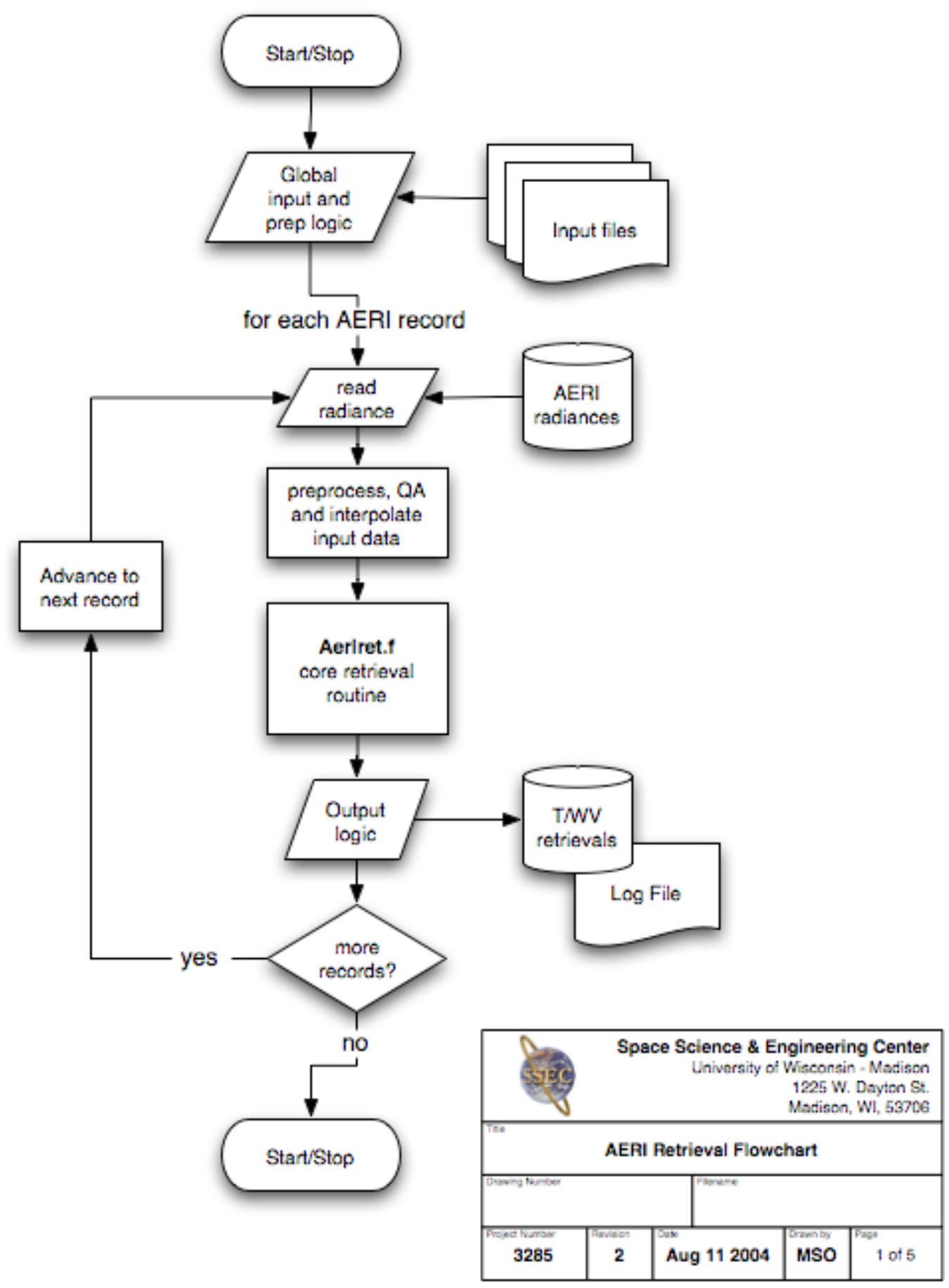

E. 1 
AERI retrieval software flow charts - 2004

2. Global Input \& preparation logic

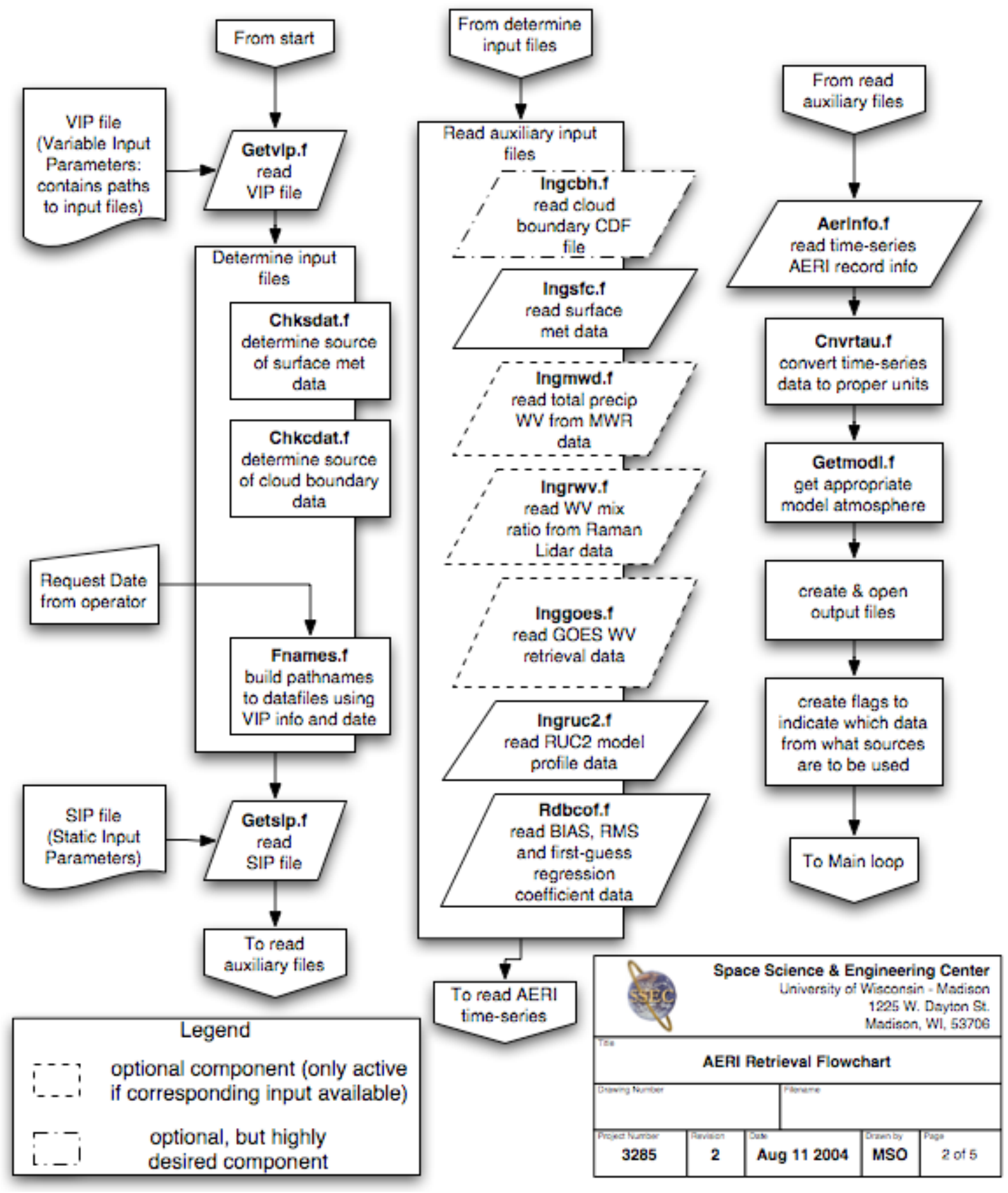


AERI retrieval software flow charts - 2004

3. Preprocess, QA radiances, interpolate auxiliary data

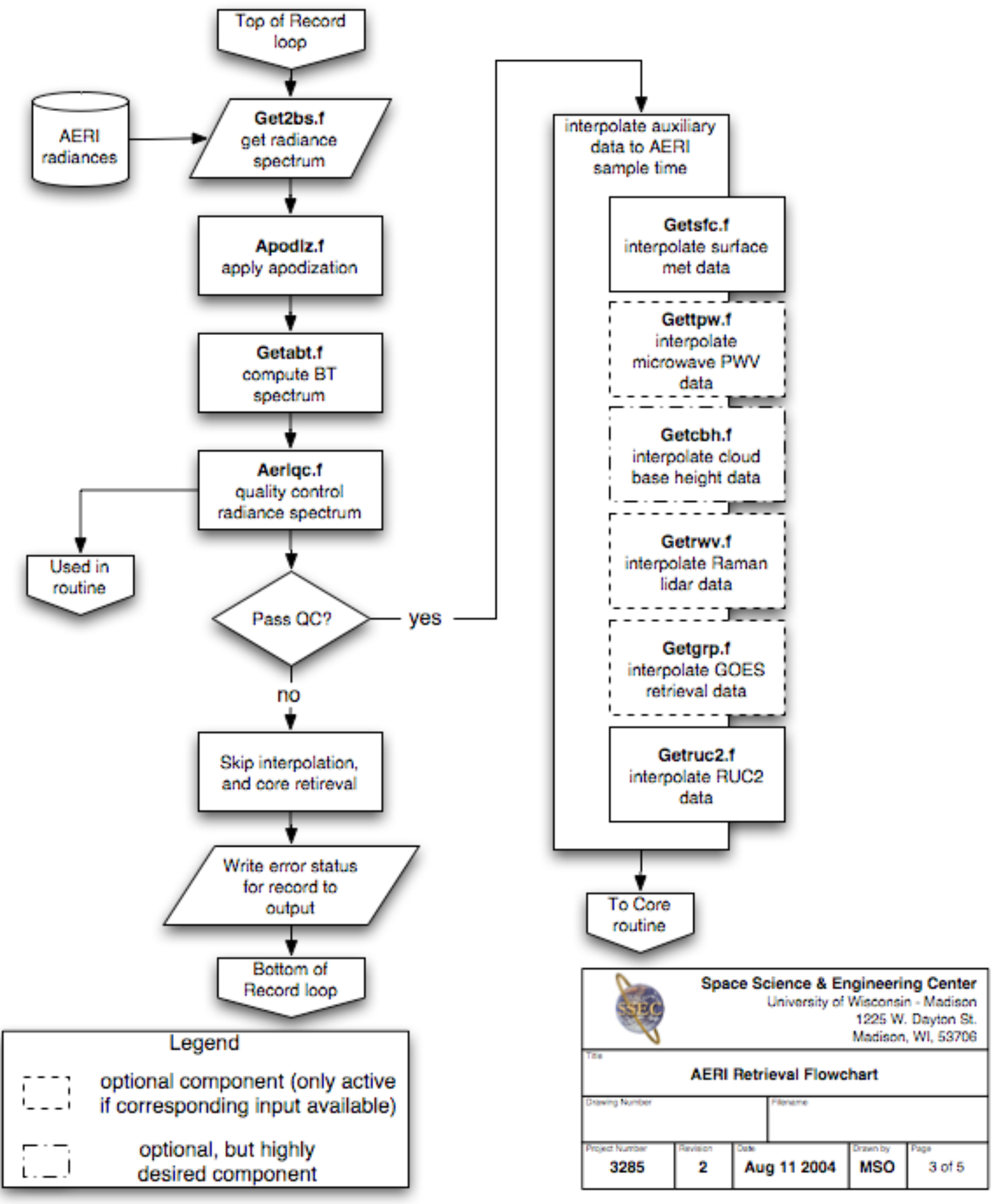


AERI retrieval software flow charts - 2004

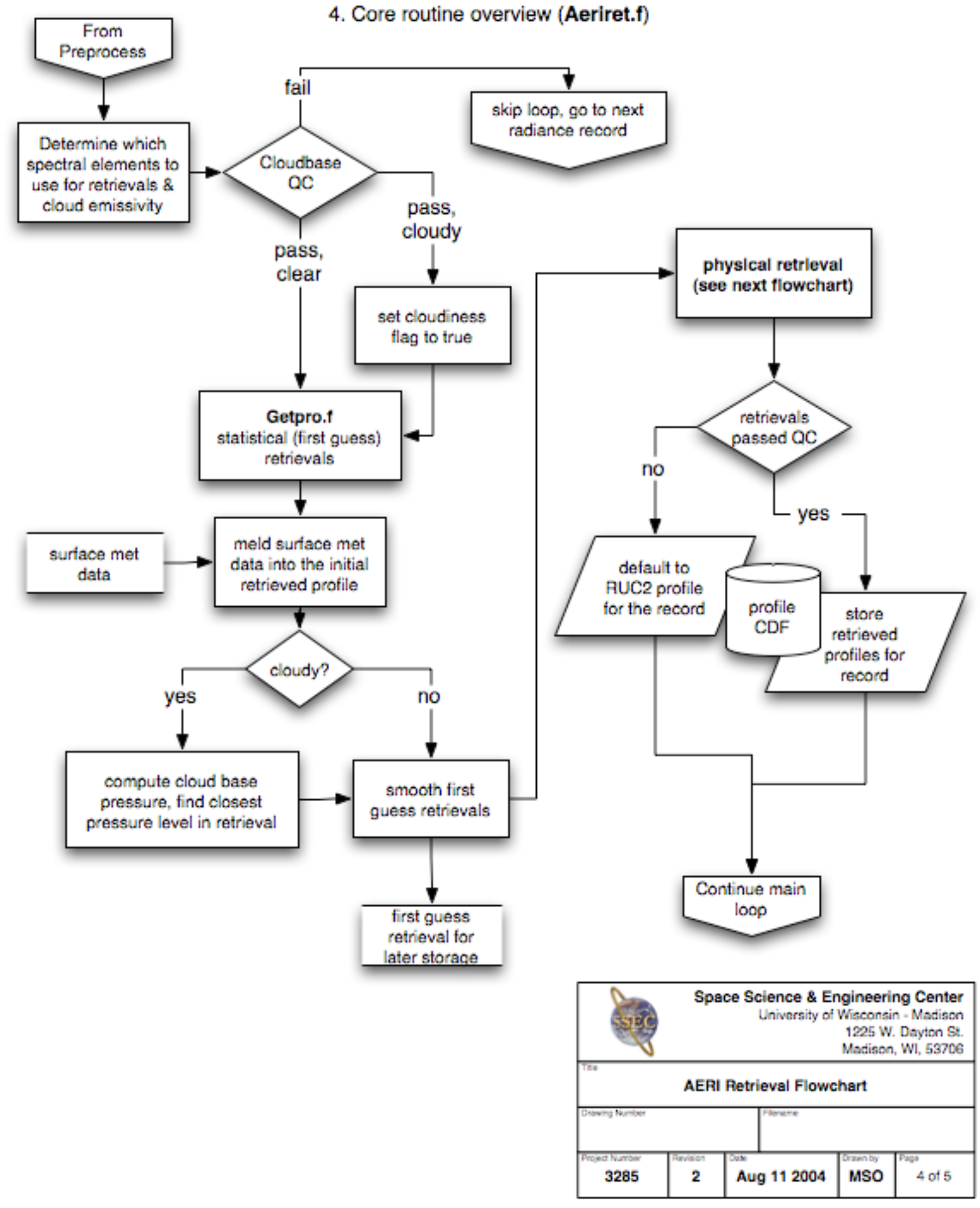


AERI retrieval software flow charts - 2004

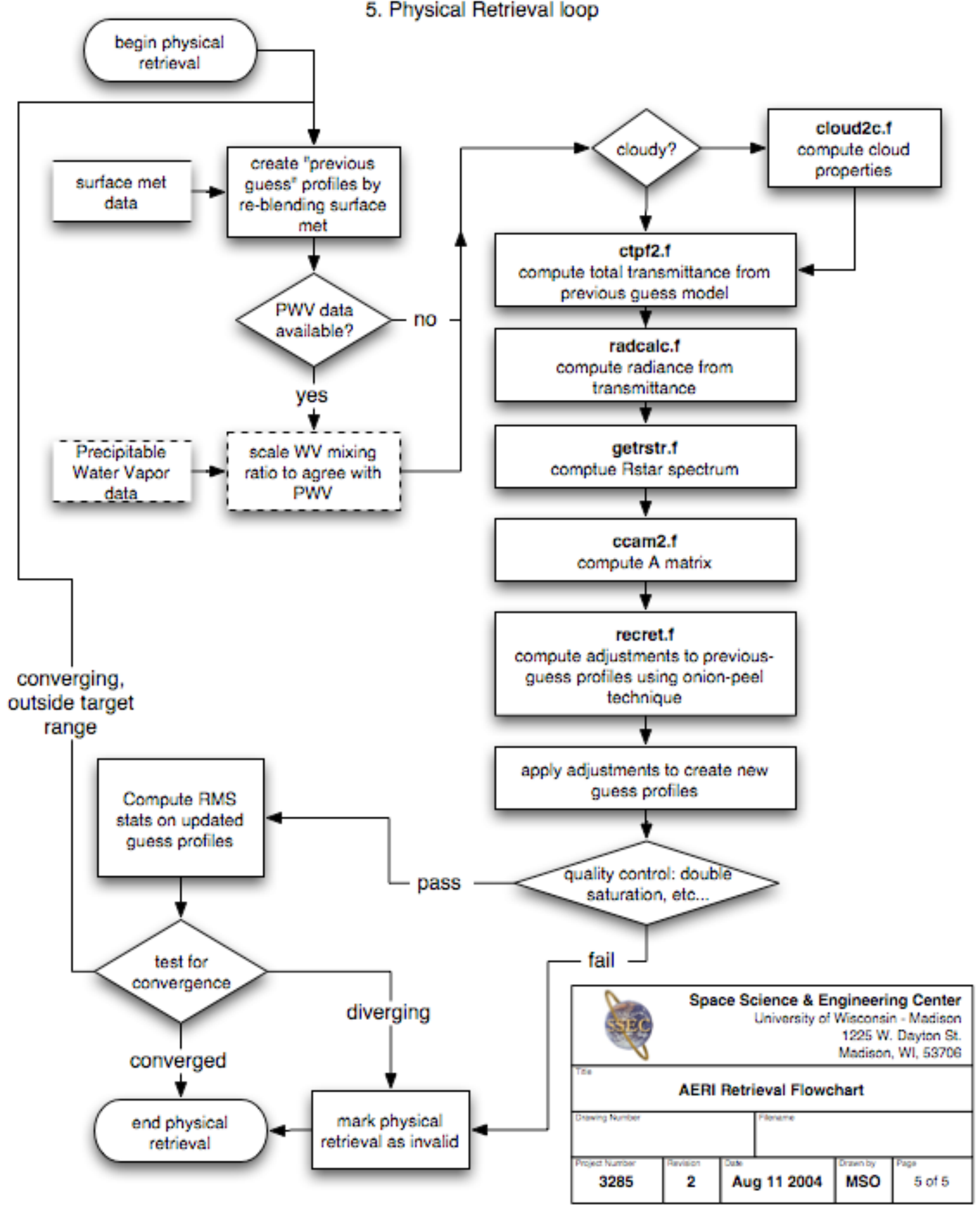

